\title{
GENERIC REPRESENTATIONS ARE INDUCED FROM SQUARE-INTEGRABLE REPRESENTATIONS
}

\author{
BY
}

RONALD L. LIPSMAN ${ }^{1}$

\begin{abstract}
It is proven for arbitrary real algebraic groups that the generic irreducible unitary representation is induced from a square-integrable representation (modulo the projective kernel). This generalizes the well-known result for reductive groups that the generic representations are either discrete series, or induced from discrete series (modulo the nilradical) representations of cuspidal parabolic subgroups.
\end{abstract}

1. Introduction. In this paper we shall prove the following result.

THEOREM A. Let $G$ be a real algebraic group. Then, with the exception of a Plancherel null set, every irreducible unitary representation of $G$ has the property that it is induced (from an algebraic subgroup) by a representation which is squareintegrable modulo its projective kernel.

The motivation for this result is the following. The Orbit Method, which has had a flourishing development in recent years (see e.g., $[2,3,8,9,14])$, has as its basic philosophy that harmonic analyses of all Lie groups have many features in common. Indeed, many strong similarities have been discovered between the construction and parametrization of the irreducible unitary representations, the characters and Plancherel measure of reductive groups on one hand and solvable groups on the other. Along these lines many classical results about semisimple groups have been reformulated in the orbit language, which developed originally in the solvable theory. Perhaps [14] is the most famous instance of this development. In this paper, I should like to reverse the roles, i.e. I will formulate a new result for solvable groups-indeed for general groups-based on a classical part of the semisimple theory. Namely, one of the most important results about semisimple groups is the description of the generic representations as members of several series. They are either discrete series, or various principal series, the latter being induced representations from cuspidal parabolics by representations which are trivial on the unipotent radical and discrete series on a Levi factor. This can be summarized by saying that the generic representations are induced by representations squareintegrable modulo their projective kernel. Theorem A asserts that this remains true for any algebraic group. This result is already known for nilpotent groups [13]. Actually it follows just from the existence of real polarizations. But Penney's result is stronger, since his inducing subgroup is canonical (and much larger than a polarization). One can also show the existence of a canonical inducing subgroup for

Received by the editors October 30, 1983 and, in revised form, December 13, 1983.

1980 Mathematics Subject Classification. Primary 22E45; Secondary 20 G05.

${ }^{1}$ Research supported by NSF grant MCS 82-00706. 
reductive groups of the Harish-Chandra class. It is an interesting question (which I hope to return to) as to whether such canonical groups always exist.

I would like to express my gratitude to Michel Duflo and Roger Howe. Duflo first pointed out to me examples like those in Subsection 4a. But it was Howe who strongly concurred with my original sentiment about the truth of Theorem A, and who helped me over one or two rough spots in several letters and conversations.

2. Notation and terminology. $G$ will denote a real algebraic group, that is $G=\underline{G}(\mathbf{R})$, where $\underline{G}$ is a (complex) algebraic group def $/ \mathbf{R} . G$ is, in particular, a real Lie group. The Lie algebra of $G$ is denoted by $\mathfrak{g}$, and its real dual by $\mathfrak{g}^{*}$. We consider the set $A P(G)$ of admissible, well-polarizable linear functionals $\phi \in \mathfrak{g}^{*}$. The precise definition of the set $A P(G)$ may be found in [2 or 9], but the reader should think of its elements as "regular" and "integral" linear functionals. Corresponding to each $\phi \in A \mathcal{A}(G)$ there is associated a canonical finite set $\mathfrak{X}_{G}^{i}(\phi)$ of irreducible unitary representations of a 2-fold cover of the stability group $G_{\phi}$; see $\left[2,9\right.$ or 10]. $\left(\mathfrak{X}_{G}^{i}(\phi)\right.$ is naturally identified to the projective dual of $G_{\phi} / G_{\phi}^{0}$ with a canonical 2-cocycle determined by $\phi$.) We set

$$
B(G)=\left\{(\phi, \tau): \phi \in \mathcal{A P}(G), \tau \in \mathfrak{X}_{G}^{i}(\phi)\right\} .
$$

$B(G)$ is a $G$-space in a natural way. In [2] Duflo has constructed a map $(\phi, \tau) \rightarrow$ $\pi(\phi, \tau)$ from $B(G)$ to the dual $\hat{G}$ of equivalence classes of irreducible unitary representations of $G$. The map factors to an injection $B(G) / G \rightarrow \hat{G}$. The image consists of generic classes in the sense that its complement is of null Plancherel measure. (Since algebraic groups are automatically type I, the Plancherel measure class is well defined.) Duflo's construction of the class $\pi(\phi, \tau)$ is by induction on the dimension of $G$. Explicit realizations of the representations by harmonic induction via polarizations is taken up in [9 and 11]. In this ppaer we show that there is a Plancherel co-null set in $\hat{G}$, contained in $B(G) / G$, whose classes have the property that they can be realized by ordinary induction by a representation which is square-integrable modulo its projective kernel.

Here is some more mundane notation and terminology. $G^{0}$ will denote the identity component (for the locally compact topology of $G$ ), $Z_{G}$ denotes the center of $G$. More generally, if $V$ is a $G$-space and $\mathcal{W}$ is a subset of $V$, then

$$
Z_{G}(\mathcal{W})=\{g \in G: g \cdot w=w \forall w \in \mathcal{W}\} .
$$

We also write $N_{G}(\mathcal{W})$ for the group elements that preserve $\mathcal{W}$ set-wise, and $G_{v}$ for the stability group of an element $v \in V$. If $\pi$ is an irreducible unitary representation of $G$, we put $K_{\pi}=\operatorname{ker} \pi$ and $P_{\pi}=\operatorname{proj} \operatorname{ker} \pi=\{g \in G: \pi(g)$ is a scalar operator $\}$. Such a representation $\pi$ is called (resp.) square-integrable, square-integrable mod its center, or square-integrable mod its projective kernel, if there exists a matrix coefficient of $\pi$ which is square-integrable (resp.) on $G, G / Z_{G}$ or $G / P_{\pi}$. These are equivalent to the occurrence of $\pi$ as a discrete summand in (resp.) the regular representation of $G$, the representation $\operatorname{Ind}_{Z_{G}}^{G} \chi$, or $\operatorname{Ind}_{P_{\pi}}^{G} \chi$, where $\left.\pi\right|_{P_{\pi}}=\chi$ Id. We remark that $\pi$ is square-integrable $\bmod P_{\pi} \Leftrightarrow \pi$, considered as a representation of $G / K_{\pi}$, is square-integrable mod its center. Finally, by a split torus $A$ in $G$ we shall mean $A=\underline{A}(\mathbf{R})^{0}$, where $\underline{A}$ is a split (over $\mathbf{R}$ ) torus in $\underline{G}$ in the usual sense. Such a group is a vector group. The word torus by itself indicates a connected compact abelian Lie group. 
3. The main result. The following is the main result of the paper.

THEOREM A. Let $G$ be a real algebraic group. Then, with the exception of a Plancherel null set, every irreducible unitary representation of $G$ has the property that it is induced (from an algebraic subgroup) by a representation which is squareintegrable modulo its projective kernel.

We call a representation with the property of Theorem A square-integrably induced. The proof of Theorem A occupies this entire section, and proceeds by a series of steps which we begin now.

3a. Reductive data. The following material is adapted from $[11, \S 4]$. Let $(\phi, \tau) \in$ $B(G), \pi=\pi(\phi, \tau)$. We let $N$ be the unipotent radical of $G, \mathfrak{n}$ its Lie algebra, $\theta=\left.\phi\right|_{\mathfrak{n}}$. We set $G^{1}=G_{\theta} N, \phi^{1}=\left.\phi\right|_{\mathfrak{g}^{1}}$. Then by $[11$, Proposition 4.1 , we have $\phi^{1} \in A \mathcal{P}\left(G^{1}\right)$ and there is an element $\tau^{1} \in \mathfrak{X}_{G^{1}}^{i}\left(\phi^{1}\right)$, canonically determined by $\tau$, such that

$$
\pi(\phi, \tau)=\operatorname{Ind}_{G^{1}}^{G} \pi_{G^{1}}\left(\phi^{1}, \tau^{1}\right) .
$$

We repeat the procedure with $\left(\phi^{1}, \tau^{1}\right) \in B\left(G^{1}\right)$. Let $N^{1}$ be the unipotent radical of $G^{1}, \theta^{1}=\left.\phi^{1}\right|_{\mathfrak{n}^{1}}, \ldots$. The sequence stabilizes after a finite number of steps, say $r$,

$$
G^{r}=\left(G^{r}\right)_{\theta^{r}} N^{r}, \quad\left(\phi^{r}, \tau^{r}\right) \in B\left(G^{r}\right) ;
$$

and by induction in stages we obtain

$$
\pi(\phi, \tau)=\operatorname{Ind}_{G^{r}}^{G} \pi_{G^{r}}\left(\phi^{r}, \tau^{r}\right) .
$$

Let $\gamma^{r}=\pi_{N^{r}}\left(\theta^{r}\right)$ be the Kirillov representation of $N^{r}$ determined by $\theta^{r}$. Then select a Levi factor $S$ of $G^{r}$ which lies in $\left(G^{r}\right)_{\theta^{r}}, G^{r}=S N^{r}$. Since $S$ fixes $\theta^{r}, \gamma$ extends canonically to a (perhaps projective) representation $\tilde{\gamma}$ of $S$ on the space of $\gamma[\mathbf{1}]$. Thus (by [12]) there is canonically determined a (perhaps projective) irreducible unitary representation $\omega$ of $S$ such that

$$
\pi\left(\phi^{r}, \tau^{r}\right) \equiv \omega \otimes \tilde{\gamma} \times \gamma .
$$

We call $(S, \omega)$ the reductive data of $\pi$, or of $(\phi, \tau)$. We know that the order of the cocycle for the representation $\omega$ is at most two. (These data are uniquely determined only up to conjugation by certain elements of $N^{r}$, but that need not concern us.)

By the above construction and an argument to be given in subsection $3 \mathrm{~d}$, we will see it is enough to consider groups of the type $G^{r}$. The first step in that direction is the case of trivial unipotent radical. We take that up in the next subsection.

3b. Reductive groups. We shall prove Theorem A in this subsection under the assumption that $G$ is reductive. If $G$ is connected, or more generally of the HarishChandra class, then of course Theorem A is well known and, as discussed in the introduction, is the inspiration for the result in general. The inducing subgroups are then cuspidal parabolics, and the representations $\pi(\phi, \tau)$ are square-integrably induced for every $(\phi, \tau) \in B(G)$. But if $G$ is not of the Harish-Chandra class, the stability group $G_{\phi}$ may not leave any cuspidal parabolic subalgebra invariant. That is because it may mix up the root spaces extensively if the action of $G_{\phi}$ on the Cartan subalgebra $\mathfrak{g}_{\phi}$ is too complicated. But in that case, it follows that generically the stability group in $G_{\phi} / G_{\phi}^{0}$ for the action on $\left(G_{\phi}^{0}\right)^{-}$must be quite small. This line of thought may be pursued to show that generically one is able 
to realize the representations of $G$ by inducing from (essentially) Harish-Chandra class subgroups.

We begin with the simple-but instructive case that $G^{0}$ is abelian. Let $G$ be a reductive algebraic group, and suppose $\mathfrak{g}$ is abelian. What does it mean for $G$ to be of the Harish-Chandra class? The key defining condition (for a reductive group) of the Harish-Chandra class is: $\operatorname{Ad}_{\mathfrak{g}_{c}} G \subseteq \operatorname{Ad} \mathfrak{g}_{c}$. If $\mathfrak{g}$ is abelian, that means $G$ acts trivially on $\mathfrak{g}$, or equivalently $G^{0}$ is central in $G$. For our purposes it will be enough to arrange that a maximal split torus of $G^{0}$ is centralized by $G$. Here is the key

LEMMA B. Let $F$ be a finite group of automorphisms of a vector group $V$. Then there exists $U \subseteq \hat{V}$, an open co-null subset, such that

$$
F_{\chi}=Z_{F}(V), \quad \forall \chi \in \mathcal{U} .
$$

Proof. Let us write $\Phi: F \rightarrow \operatorname{Aut}(V)$ to denote the action of $F$ on $V$. Then the lemma asserts the existence of an open co-null subset $\mathcal{U}$ in $\hat{V}$ such that $F_{\chi}=\operatorname{Ker} \Phi$ for all $\chi \in \mathcal{U}$. (Note: $F$ acts on $\hat{V}$ by duality $(f \cdot \chi)(v)=\chi\left(f^{-1} \cdot v\right), v \in V, \chi \in \hat{V}$.) This can be proven rather easily by appealing to theorems on principal orbit types for actions of compact groups. Here is an absolutely elementary proof due to $\mathrm{N}$. Markley.

Let $\mathcal{W}=\left\{\chi \in \hat{V}: \#\left(F_{\chi}\right)\right.$ is minimum $\} . \mathcal{W}$ is open and co-null. List the stability groups that occur in $\mathcal{W}$, say $F_{1}, F_{2}, \ldots, F_{r}$. Let $\mathcal{W}_{i}=\left\{\chi \in \mathcal{W}: F_{\chi}=F_{i}\right\}, 1 \leq i \leq r$. Then $\mathcal{W}_{i}$ is closed in $\mathcal{W}$. Indeed, if $\chi_{n} \in \mathcal{W}_{i}$ and $\chi_{n} \rightarrow \chi \in \mathcal{W}$, then $F_{\chi} \supseteq F_{i}$. Since $\#\left(F_{\chi}\right)=\#\left(F_{i}\right)$, we get $\chi \in \mathcal{W}_{i}$. But $\mathcal{W}$ is a disjoint union of the $\mathcal{W}_{i}$; hence each $\mathcal{W}_{i}$ is open in $\hat{V}$. Now a linear automorphism of a (real) vector space that pointwise fixes a nonempty open set must be the identity. Hence $F_{\chi} \subseteq Z_{F}(\hat{V}) \forall \chi \in W$. By duality we obtain $F_{\chi} \subseteq Z_{F}(V), \forall \chi \in \mathcal{W}$. The reverse inclusion is obvious, so the lemma is proven.

How do we use the lemma to prove Theorem A for reductive algebraic groups with abelian Lie algebra? Let $G^{0}$ be the identity component of $G, T$ the maximal torus of $G^{0}$. Let $F=\operatorname{Ad}_{\mathfrak{g}} G$. Then $T$ is $F$-invariant. Choose an $F$-invariant complement $\mathfrak{v}$ to $\mathfrak{t}$ in $\mathfrak{g}$. Then $V=\exp \mathfrak{v}$ is a closed $G$-invariant vector subgroup of $G^{0}$. Apply Lemma B: $\exists \mathcal{U} \subseteq \hat{V}$, co-null, such that $G_{\chi}=Z_{G}(V), \forall \chi \in \mathcal{U}$. The representations of $G$ that lie over $\mathcal{U}$ constitute a Plancherel co-null set and every one of them is of the form

$$
\pi=\operatorname{Ind}_{H}^{G} \sigma
$$

where $H=Z_{G}(V), \sigma \in \hat{H},\left.\sigma\right|_{V}=$ multiple of some $\chi \in \mathcal{U}$. It is obvious that $\sigma$ is finite dimensional and that $H / Z_{H}$ is compact (since $V \subseteq Z_{H}$ ). In particular, $\sigma$ is square-integrable mod its projective kernel (even mod its center).

Now we extend to arbitrary reductive algebraic groups $G$. Fix a Cartan subalgebra $\mathfrak{h}$ of $\mathfrak{g}$. We shall only consider $\phi \in A \mathcal{P}(G)$ such that $\mathfrak{g}_{\phi}=\mathfrak{h}$. Let $T$ be the maximal torus in the abelian group $H=G_{\phi}^{0}$. Set $F=N_{G}(\mathfrak{h}) / H$, and note that $G_{\phi} \subseteq N_{G}(\mathfrak{h}) . T$ is invariant under $F$, so we can choose an $F$-invariant complement $\mathfrak{a}$ in $\mathfrak{h}$. Set $A=\exp \mathfrak{a}$ and apply Lemma B: $\exists U \subseteq \hat{A}$, co-null, such that $A$ is central in $N_{G}(\mathfrak{h})_{\chi}, \chi \in \mathcal{U}$. Now consider the representations $\pi(\phi, \tau)$ for which $\left.i \phi\right|_{\mathfrak{a}} \in \log \mathcal{U}$. Their complement in the part of $\hat{G}$ associated to $\mathfrak{h}$ is a null set-and if we vary over a set of conjugacy classes of Cartan subalgebras, these exhaust a set, co-null with respect to Plancherel measure on $\hat{G}$. So it is enough to show that 
every $\pi(\phi, \tau),\left.i \phi\right|_{\mathfrak{a}} \in \log \mathcal{U}$, is square-integrably induced. (Note: if $\left.i \phi\right|_{\mathfrak{a}} \in \log \mathcal{U}$, then $A$ is centralized by $G_{\phi}$.)

We realize $\pi=\pi(\phi, \tau)$ as a group extension representation over $G^{0}$. There is $\tau^{0} \in \mathfrak{X}_{G^{0}}^{i}(\phi)$ such that $\pi$ lies over $\pi^{0}=\pi_{G^{0}}\left(\phi, \tau^{0}\right)$. Moreover, $\pi^{0}$ is induced from a cuspidal parabolic subgroup of $G^{0}$ which we can specify as follows (see [2, III.3; 9, $\S 4 \mathrm{c}])$. Let ${ }^{0} M \equiv Z_{G^{0}}(\mathfrak{a})$ and choose $X \in \mathfrak{a}$ such that if a root $\alpha$ of $\left(\mathfrak{g}_{c}, \mathfrak{h}_{c}\right)$ satisfies $\alpha(X)=0$, then $\alpha(\mathfrak{a})=0$. Set $\mathfrak{u}=\left(\sum_{\alpha(X)>0} \mathfrak{g}^{\alpha}\right) \cap \mathfrak{g}, U=\exp \mathfrak{u}$. Then ${ }^{0} P={ }^{0} M U$ is a cuspidal parabolic subgroup of $G^{0}$ and $\pi^{0}=\operatorname{Ind}_{0}^{G} \sigma$, where $\sigma(U)=1$, and $\left.\sigma\right|_{0_{M}}$ is the square-integrable representation $\bmod Z_{{ }} M$ associated canonically to the data $\left(\left.\phi\right|_{\mathfrak{m}}, \tau^{0}\right) . \sigma$ is obviously square-integrable mod its projective kernel. Moreover, $\sigma$ (resp. $\pi^{0}$ ) can be realized by harmonic induction from $\tau^{0}$ on $\left(G^{0}\right)_{\phi}=\left({ }^{0} M\right)_{\phi \mid \mathfrak{m}}$ via a polarization for $\phi \mid \mathfrak{m}$ (resp. $\left.\phi\right)$ which is $\left(G^{0}\right)_{\phi}$-invariant. Now what is the stability group $G_{\pi^{0}}$ of $\pi^{0}$ in $G$ ? Clearly it is $\left(G_{\phi}\right)_{\tau^{0}} G^{0} \subseteq G_{\phi} G^{0}$. The only thing to be shown therefore is that any extension of $\pi^{0}$ to $\left(G_{\phi}\right)_{\tau^{0}} G^{0}$ is still square-integrably induced. The point is that the polarization for $\phi$ which gives $\pi^{0}$ can be chosen $G_{\phi}$-invariant (e.g. because $G_{\phi}$ preserves the set of imaginary roots which are positive with respect to $\left.\phi\right|_{\mathfrak{t}}$, and $G_{\phi}$ centralizes $\mathfrak{a}$, so $X$ ). So $\pi$ is also harmonically induced (this time from $\tau$ on $G_{\phi}$ ) by that polarization. More precisely, using the technique of $[9, \S 4 \mathrm{c}]$, we see that if we set $P={ }^{0} P G_{\phi}$, then the representation $\pi$ is induced from $P$ by a representation which is trivial on $U$, and on $M={ }^{0} M G_{\phi}$ is given by harmonic induction from $\tau$ on $G_{\phi}$. The latter is square-integrable $\bmod Z_{M}$. So $\pi$ is square-integrably induced. That completes the proof of Theorem A for reductive algebraic groups.

Let us summarize: If $G$ is reductive algebraic, then aside from a Plancherel null set every $\pi \in \hat{G}$ is induced from a parabolic-type subgroup $P=M U$ by a representation $\sigma$ which satisfies $\sigma(U)=1, \sigma$ is square-integrable $\bmod Z_{M}$, and there is a maximal split torus of $Z_{M^{0}}$ which is contained in $Z_{M}$. When the maximal split torus is central in $G^{0}$ (i.e. the discrete series), then $U=\{1\}$ and $M=G_{\phi} G^{0}$ is of finite index in $G$ (e.g. when $\mathfrak{g}$ is abelian).

3c. Semidirect products. Now consider a group $G=S N$ with $N$ the unipotent radical, $S$ reductive, $\phi \in A P(G), \theta=\left.\phi\right|_{\mathfrak{n}}$, and $S$ fixes $\theta$. We saw in subsection 3a that any $\pi(\phi, \tau)$ is of the form $\omega \otimes \tilde{\gamma} \times \gamma, \gamma=\pi_{N}(\theta)$. By passing to a 2-fold covering if necessary, we may assume that $\omega$ is an ordinary representation of $S$ (see the comment at the end of the proof of Proposition C below). Then if $\xi=\left.\phi\right|_{\mathbf{s}}$, there is an element $\nu \in \mathfrak{X}_{S}^{i}(\xi)$, canonically determined by $\tau$, such that

$$
\pi_{G}(\phi, \tau)=\pi_{S}(\xi, \nu) \otimes \tilde{\gamma} \times \gamma
$$

(see [2]).

Proposition C. Let $\pi(\xi, \nu)$ be square-integrably induced. Then the same is true of $\pi(\phi, \tau)$.

ProOF. According to the results of subsection $3 \mathrm{~b}$, there is: (i) a parabolic type subgroup $P=M U$ of $S$ and a maximal split toral subgroup $A$ of $Z_{M^{0}}$ which is centralized by $M$; and (ii) a representation $\sigma$ of $P$ with $\sigma(U)=1$ and $\left.\sigma\right|_{M}$ squareintegrable $\bmod Z_{M}$; such that $\pi(\xi, \nu)=\operatorname{Ind}_{P}^{G} \sigma$. Now $S$, as well as any subgroup, fixes $\theta$; therefore by routine arguments we have

$$
\pi(\xi, \nu) \otimes \tilde{\gamma} \times \gamma \cong \operatorname{Ind}_{P N}^{G}(\sigma \otimes \tilde{\gamma} \times \gamma)
$$


(see e.g. [7, Lemma 3.3 and $\S 6]$, also $[9, \S 3 \mathrm{c}]$ ). So we may concentrate on the representation $\sigma \otimes \tilde{\gamma} \times \gamma$ of $P N$. The unipotent radical of the latter is $U N . M$ is a Levi factor, and the linear functional $0+\theta$ on $\mathfrak{u}+\mathfrak{n}$ is fixed by $M$. Thus, to prove Proposition $\mathrm{C}$, it will be enough to prove it under the assumption that $S=M$. We shall achieve that by employing induction on the dimension of $N$.

The cases $\operatorname{dim} N=0$ or 1 are taken care of by subsection $3 \mathrm{~b}$. Let $N^{\prime}$ be the Penney subgroup of $N$ determined by $\theta[\mathbf{1 3}] . N^{\prime}$ is canonically defined-in particular, it is invariant under any automorphism of $N$ that fixes $\theta$. Furthermore,

$$
\gamma_{N}(\theta) \cong \operatorname{Ind}_{N^{\prime}}^{N} \gamma_{N^{\prime}}\left(\theta^{\prime}\right), \quad \theta^{\prime}=\left.\theta\right|_{\mathfrak{n}^{\prime}},
$$

and $\gamma^{\prime}$ is square-integrable mod its projective kernel [13]. But much more is true. In fact (if we continue to write $\sigma=\pi(\xi, \nu) \in \hat{M}$ ), we have

$$
\sigma \otimes \tilde{\gamma} \times \gamma \cong \operatorname{Ind}_{M N^{\prime}}^{M N} \sigma \otimes \tilde{\gamma}^{\prime} \times \gamma^{\prime}
$$

(This is standard-see the same references as cited above.) Thus, by the induction assumption, we may assume $N^{\prime}=N, \operatorname{dim} Z_{N}=1$, and $\gamma$ is square-integrable mod its center. Unfortunately, however, $\sigma \otimes \tilde{\gamma} \times \gamma$ may not be square-integrable mod its center (or projective kernel). This is because the center of $M$ may not centralize $N$. In particular, $A$ may not be central in $M N$. The remainder of the proof deals with this difficulty.

Let $\mathfrak{z}=$ Cent $\mathfrak{n}$ and consider the ascending central series $\mathfrak{z} \subseteq \mathfrak{z}^{(2)} \subseteq \ldots$. Diagonalize the action of $A$ on $\mathfrak{z}^{(2)}$. Suppose there is a nontrivial eigenvalue, say

$$
\mathfrak{z}_{\alpha}^{(2)}=\left\{X \in \mathfrak{z}^{(2)}: a \cdot X=\alpha(a) X, a \in A\right\} \neq\{0\}, \quad \alpha \not \equiv 1 .
$$

Then the ideal $\mathfrak{n}_{\alpha}^{(2)}=\mathfrak{z}+\mathfrak{z}_{\alpha}^{(2)}$ is normalized by $M$ (since $A$ is central in $M$ ); so is the proper ideal $\mathfrak{n}^{\alpha}=Z_{\mathfrak{n}}\left(\mathfrak{n}_{\alpha}^{(2)}\right)$. Now the symplectic form $B_{\theta}(X, Y)=\theta[X, Y]$ is $A$-invariant and nondegenerate on $\mathfrak{n} / \mathfrak{z}$. It follows that $\mathfrak{n}_{\alpha}^{(2)}$ is abelian and contained in $\mathfrak{n}^{\alpha}$. Furthermore, the stabilizer in $\mathfrak{n}$ of $\left.\theta\right|_{\mathfrak{n}_{\alpha}^{(2)}}$ is $\mathfrak{n}^{\alpha}$. Therefore $\gamma=\operatorname{Ind}_{N^{\alpha}}^{N} \gamma^{\alpha}$, where $N^{\alpha}=\exp \mathfrak{n}^{\alpha}$, and $\gamma^{\alpha}=\pi_{N^{\alpha}}\left(\left.\theta\right|_{\mathfrak{n}^{\alpha}}\right)$ is square-integrable mod its projective kernel. Of course, we also have

$$
\sigma \otimes \tilde{\gamma} \times \gamma \cong \operatorname{Ind}_{M N^{\alpha}}^{M N} \sigma \otimes \tilde{\gamma}^{\alpha} \times \gamma^{\alpha} .
$$

The conclusion follows then by induction.

But what if there is no nontrivial eigenvalue in $z^{(2)}$ ? Then we diagonalize the action of $A$ on $\mathfrak{z}^{(3)}$; say

$$
\mathfrak{z}_{\beta}^{(3)}=\left\{X \in \mathfrak{z}^{(3)}: a \cdot X=\beta(a) X, a \in A\right\} \neq\{0\}, \quad \beta \not \equiv 1 .
$$

Unfortunately, we cannot proceed exactly as with $\mathfrak{z}^{(2)}$. Although $\mathfrak{z}_{\beta}^{(3)}+\mathfrak{z}$ is an algebra (since $\left[\mathfrak{z}_{\beta}^{(3)}, \mathfrak{z}_{\beta}^{(3)}\right]=0$; indeed, it is in $\mathfrak{z}^{(2)}$ which is fixed by $A$, so if $X, Y \in$ $\mathfrak{z}_{\beta}^{(3)}$, then $\left.[X, Y]=a \cdot[X, Y]=\beta^{2}(a)[X, Y] \Rightarrow[X, Y]=0\right)$, it is not an ideal. Instead we take $\mathfrak{n}_{\beta}^{(3)}=\mathfrak{z}^{(2)}+\mathfrak{z}_{\beta}^{(3)}$. This is obviously an ideal, but it may not be abelian. Set $\theta_{\beta}=\left.\theta\right|_{\mathfrak{n}_{\beta}^{(3)}}, N_{\beta}=\exp \mathfrak{n}_{\beta}^{(3)}$ and $\gamma_{\beta}=\pi_{N_{\beta}}\left(\theta_{\beta}\right)$. Claim: The stabilizer $N^{\beta}$ in $N$ of $\gamma_{\beta}$ is a subgroup of positive codimension. Well, since $\gamma$ is square-integrable $\bmod Z_{N}$, we know $N \cdot \theta=\theta+\mathfrak{z}^{\perp}$. Therefore $\operatorname{dim} N \cdot \theta_{\beta}=\operatorname{dim} \mathfrak{n}_{\beta}^{(3)}-1$. But 
$\operatorname{dim} N_{\beta} \cdot \theta_{\beta} \leq \operatorname{dim} \mathfrak{n}_{\beta}^{(3)}-2$. This is because the group $Z_{N} \exp \mathfrak{z}_{\beta}^{(3)}$ does not act on $\theta_{\beta}$. This is clear for $Z_{N}$. Regarding $\exp \mathfrak{z}_{\beta}^{(3)}$, we have $\theta\left[\mathfrak{z}_{\beta}^{(3)}, \mathfrak{n}_{\beta}^{(3)}\right]=0$ because: first of all, $\left[\mathfrak{z}_{\beta}^{(3)}, \mathfrak{z}_{\beta}^{(3)}\right]=0$ (see above); and, second, if $X \in \mathfrak{z}^{(2)}, Y \in \mathfrak{z}_{\beta}^{(3)}$ and $\theta[X, Y] \neq 0$, then $\beta(a) \theta[X, Y]=\theta[X, a \cdot Y]=a \cdot \theta\left[a^{-1} \cdot X, Y\right]=\theta\left[a^{-1} \cdot X, Y\right]=\theta[X, Y](A$ fixes $\mathbf{z}^{(2)}$.) By standard Mackey and Kirillov theory, we know that $N^{\beta}=\left\{n \in N: n \cdot \theta_{\beta} \in\right.$ $\left.N_{\beta} \cdot \theta_{\beta}\right\}$. Hence (because $\operatorname{dim} N \cdot \theta_{\beta}>\operatorname{dim} N_{\beta} \cdot \theta_{\beta}$ ) we see that $N^{\beta}$ is of positive codimension in $N$. Of course, $\gamma=\operatorname{Ind}_{N^{\beta}}^{N} \pi_{N^{\beta}}\left(\left.\theta\right|_{\mathfrak{n}^{\beta}}\right)$. Since $M$ fixes $\theta$ and $\mathfrak{n}_{\beta}^{(3)}$, we are once again in a position to apply the induction hypothesis. Thus either we are done or $A$ fixes $\mathfrak{z}^{(3)}$. It is clear that by continuing up the ascending central series either we conclude the argument by induction or we find that there are no nontrivial eigenvalues for the action of $A$ on $\mathfrak{n}$. In that case $A$ is central in all of $M N$, and the representation $\sigma \otimes \tilde{\gamma} \times \gamma$ is square-integrable mod its center.

We remark finally that if $\omega$ is a projective - not ordinary-representation, and we had to pass to a 2 -fold cover $\tilde{S}$ of $S$, then the final result is of the form $\pi(\phi, \tau)=\operatorname{Ind}_{\tilde{M} N^{\prime \prime}}^{\tilde{S} N} \sigma \otimes \tilde{\gamma}^{\prime \prime} \times \gamma^{\prime \prime}$, where $\sigma \otimes \tilde{\gamma}^{\prime \prime} \times \gamma^{\prime \prime}$ is square-integrable mod its center. Furthermore, $\sigma \otimes \tilde{\gamma}^{\prime \prime}$ is trivial on the 2-element central subgroup defining the extensions of both $M$ and $S$. Hence $\pi(\phi, \tau) \cong \operatorname{Ind}_{M N^{\prime \prime}}^{S N} \sigma \otimes \tilde{\gamma}^{\prime \prime} \times \gamma^{\prime \prime}$. This completes the proof of Proposition C.

REMARK. Roger Howe has shown me an alternate proof of Proposition C. In his scheme, one replaces the Penney subgroup by canonical subquotients which are Heisenberg groups [4]. Then the ascending central series is 'rather short'! One can replace my diagonalization argument by something similar to [5, pp. 43 ff.]. Howe's argument is somewhat easier than that given above. However, the inducing subgroups which emerge from his argument are rather small (think of a real polarization rather than a Penney subgroup).

3d. Conclusion. It remains to combine the results of the preceding subsections in order to arrive at a proof of Theorem A. First of all, if there is no unipotent radical, then the theorem is true by the results of subsection $3 \mathrm{~b}$. So we may assume that $G$ has nontrivial unipotent radical $N$. We use the Plancherel theory for group extensions [6]. It is enough to show that for every $\theta \in \mathfrak{n}^{*}$, almost all representations of $G$ lying over $\gamma=\pi_{N}(\theta)$ are square-integrably induced. Equivalently it is enough to substantiate the property for almost all representations that occur in $\operatorname{Ind}_{N}^{G} \gamma$. But, by [6], that is equivalent to proving the same thing for the representation $\operatorname{Ind}_{N}^{G^{1}} \gamma$. Hence it is enough to show that for every $\theta^{1} \in\left(\mathfrak{n}^{1}\right)^{*},\left.\theta^{1}\right|_{\mathfrak{n}}=\theta$, the property is true for almost all representations that lie over $\gamma^{1}=\pi_{N^{1}}\left(\theta^{1}\right)$. That is, we need to show that almost all representations in $\operatorname{Ind}_{N^{1}}^{G^{1}} \gamma^{1}$ are square-integrably induced. Continuing in this way, we see that the proof of Theorem $\mathrm{A}$ is reduced to showing that almost every constituent of $\operatorname{Ind}_{N^{r}}^{G^{r}} \gamma^{r}$ is square-integrably induced. But that follows immediately from Proposition $\mathrm{C}$ and the work in subsection $3 \mathrm{~b}$. The argument is therefore complete and Theorem $\mathrm{A}$ is now proven.

REMARK. It is easy to trace back through the proof to see that in every instance the inducing subgroup is algebraic. We leave that chore to the reader.

4. Examples. In this section we illustrate Theorem A with two examples. The first is a reductive algebraic group $G$ not of the Harish-Chandra class. We exhibit $\phi \in A P(G)$ which do not possess invariant metric polarizations. Thus the 
corresponding representations $\pi(\phi, \tau)$, for any $\tau \in \mathfrak{X}_{G}^{i}(\phi)$, are not square-integrably induced (from parabolic-type subgroups). Of course, by Theorem A the set of such $\phi$ contributes nothing to Plancherel measure. The second example is that of a disconnected solvable group which exhibits similar behavior. One can construct more complicated semidirect products with the same kind of features, but nothing beyond the phenomena present in the two examples below is involved.

4a. A non-Harish-Chandra class reductive group. Let $\mathfrak{g}$ be the Lie algebra of $\mathrm{SL}(2, \mathrm{C})$, considered as a real Lie algebra. Consider the linear functionals $\phi \in \mathfrak{g}^{*}$ defined by

$$
\phi\left(\begin{array}{cc}
a & b \\
c & -a
\end{array}\right)=\operatorname{Re}(a \bar{\alpha})=a_{1} \alpha_{1}+a_{2} \alpha_{2}, \quad a=a_{1}+i a_{2}, \quad \alpha=\alpha_{1}+i \alpha_{2} \in \mathbf{C} .
$$

We assume $\phi \not \equiv 0$, that is $\alpha \neq 0$. Then one computes readily that $\mathfrak{g}_{\phi}=\mathfrak{h}=$ $\left\{\left(\begin{array}{cc}a & 0 \\ 0 & -a\end{array}\right): a \in \mathbf{C}\right\}$. All such $\phi$ are well polarizable. Admissibility of $\phi$ amounts to an integrability condition on $\alpha_{2}$ which we shall not specify precisely.

Now let $\varepsilon$ be the automorphism of $\operatorname{SL}(2, \mathrm{C})$ defined by

$$
\varepsilon(g)={ }^{t} \bar{g}^{-1}, \quad g \in \mathrm{SL}(2, \mathbf{C}) .
$$

Let $G$ be the corresponding semidirect product $G=\{\varepsilon\} \cdot \operatorname{SL}(2, \mathbf{C}) . \quad(\{\varepsilon\}$ denotes the 2-element group generated by $\varepsilon$.) Note that $\varepsilon$ preserves the Cartan subalgebra $\mathfrak{h}$. Also, $\varepsilon$ fixes the linear functional $\phi$ iff $\alpha_{1}=0$. Hence

$$
G_{\phi}=\left\{\begin{array}{ll}
H, & \alpha_{1} \neq 0, \\
\{\varepsilon\} H, & \alpha_{1}=0,
\end{array} \quad H=\exp \mathfrak{h}=\left\{\left(\begin{array}{cc}
a & 0 \\
0 & a^{-1}
\end{array}\right): a \in \mathbf{C}^{*}\right\} .\right.
$$

But there is no parabolic subalgebra of $\mathfrak{g}$ which is invariant under $\varepsilon$. It follows that the representations $\pi(\phi, \tau), \alpha_{1}=0$, are not square-integrably induced. The representations corresponding to $\alpha_{1} \neq 0$ on the other hand are square-integrably induced-e.g. from $P=\left\{\left(\begin{array}{ll}u & v \\ 0 & w\end{array}\right): u w \neq 0\right\}$. Note also that-in the notation of subsection $3 \mathrm{~b}-M=G_{\phi}$ and no maximal split torus of $H$ is central in $M$ if $\alpha_{1}=0$.

4b. A disconnected solvable group. Let $\mathfrak{g}$ be the split oscillator Lie algebra, i.e. the solvable Lie algebra spanned by generators $A, X, Y, Z$ satisfying commutation relations: $[A, X]=X,[A, Y]=-Y,[X, Y]=Z$. We write $G^{0}$ for the corresponding simply connected exponential solvable Lie group, and $\mathfrak{n}$ for the unipotent radical, $\mathfrak{n}=\mathbf{R} X+\mathbf{R} Y+\mathbf{R} Z$. Let $F$ be the finite cyclic group of order 4 with generator $\varepsilon$, $F=\{\varepsilon\}$. Let $G$ be the semidirect product $G=F G^{0}$, where $F$ acts on $\mathfrak{g}$ according to

$$
\varepsilon: A \rightarrow-A, X \rightarrow Y, Y \rightarrow-X, Z \rightarrow Z .
$$

In this case $A P(G)=\mathfrak{g}^{*}$. Let $\phi \in \mathfrak{g}^{*}$ be specified by $\phi=\alpha A^{*}+\xi X^{*}+\eta Y^{*}+\zeta^{*}$. Then we have

$$
\mathfrak{g}_{\phi}=\left\{\begin{array}{lc}
\mathfrak{g}, & \xi=\eta=\varsigma=0, \\
\{x X+y Y+z Z \in \mathfrak{n}: x \xi=y \eta\}, & \varsigma=0, \xi^{2}+\eta^{2} \neq 0, \\
\{a A+x X+y Y+z Z: x \xi=y \eta, a \xi=y \zeta, a \eta=x \zeta\}, \zeta \neq 0 .
\end{array}\right.
$$

Furthermore, if we write $g=\exp a A \exp x X \exp y Y \exp z Z \in G^{0}$, then

$$
g^{-1} \cdot \phi=\left(\alpha-\xi e^{a} x+\eta e^{-a} y+\varsigma x y\right) A^{*}+\left(\xi e^{a}-\varsigma y\right) X^{*}+\left(\eta e^{-a}+\varsigma x\right) Y^{*}+\varsigma Z^{*},
$$




$$
\varepsilon^{-1} \cdot \phi=-\alpha A^{*}+\eta X^{*}-\xi Y^{*}+\varsigma Z^{*} .
$$

Now we can describe the representations of $G$ as follows:

(i) $\xi=\eta=\varsigma=0$. If $\alpha=0$, we have the representations of $G$ which are trivial on $G^{0}$, i.e. the characters of $F$. If $\alpha \neq 0$, we have $G_{\phi}=\left\{\varepsilon^{2}\right\} G^{0}$ and we get two finite-dimensional representations induced by a character of $G_{\phi}$ trivial on $\exp \mathfrak{n}$.

(ii) $\varsigma=0, \xi^{2}+\eta^{2} \neq 0$. Then $G_{\phi}=\exp \mathfrak{g}_{\phi}$ and we get representations induced from $N$ by characters which are trivial on $\exp \mathbf{R} Z$.

The above representations constitute a set of Plancherel measure zero, although they are all square-integrably induced.

(iii) $\varsigma \neq 0$. We take as a cross-section for $\left\{\phi \in \mathfrak{g}^{*}: \varsigma \neq 0\right\} / G$ the set

$$
\mathcal{C}=\left\{\phi \in \mathfrak{g}^{*}: \xi=\eta=0, \varsigma \neq 0, \alpha \geq 0\right\} .
$$

Then

$$
G_{\phi}= \begin{cases}\left\{\varepsilon^{2}\right\} \exp \mathfrak{g}_{\phi}, & \alpha \neq 0 \\ \{\varepsilon\} \exp \mathfrak{g}_{\phi}, & \alpha=0 .\end{cases}
$$

If $\alpha \neq 0$, then either of the algebras $\mathfrak{h}=\mathbf{R} A+\mathbf{R} X+\mathbf{R} Z$ or $\mathfrak{h}=\mathbf{R} A+\mathbf{R} Y+$ $\mathbf{R Z}$ is a $G_{\phi^{-}}$-invariant polarization. Therefore the corresponding representations are induced from $\left\{\varepsilon^{2}\right\} H$ by characters-in particular they are square-integrably induced. However if $\alpha=0$, there does not exist a $G_{\phi^{-}}$invariant polarization for $\phi$. In particular, the four representations of $G$ that lie over $\pi_{G^{0}}(0,0,0, \zeta)$ are not squareintegrably induced. Of course (even as $\zeta$ varies) these form a set of null Plancherel measure. Finally let us match these phenomena with the proof of Theorem A. For every $\theta \in \mathfrak{n}^{*},\left.\theta\right|_{\mathfrak{z}} \neq 0, \mathfrak{z}=\mathbf{R} Z$, we have $G_{\theta} N=S N$, where $S=\{\varepsilon\} \exp \mathbf{R} A$. For $\chi_{\alpha}(\exp a A)=e^{i \alpha a}, \alpha \in \mathbf{R}$, we have: if $\alpha \neq 0$, the representations of $S$ over $\chi_{\alpha}$ are induced from the subgroup $\left\{\varepsilon^{2}\right\} \exp \mathbf{R} A$ in which the maximal split torus $\exp \mathbf{R} A$ is central; but if $\alpha=0$, the corresponding representations of $S$ are trivial on $\exp \mathbf{R} A$, i.e. are characters of $\{\varepsilon\}$, and $\exp \mathbf{R} A$ is not central in $S$.

REMARK. I close with the following two observations. It is obvious from our presentation that the property of being square-integrably induced is related to the existence of metric polarizations. The results of this paper suggest-although invariant metric polarizations may fail to exist for some $\phi \in A P(G)$-that failure is restricted to a set of Plancherel measure zero. Second, it is natural to ask whether the subgroups which induce generic irreducible representations can be chosen in a canonical fashion. Such is the case if $G$ is connected nilpotent or reductive of the Harish-Chandra class. Is it so general?

\section{REFERENCES}

1. M. Duflo, Sur les extensions des représentations irréductibles des groupes de Lie nilpotents, Ann. Sci. École Norm. Sup. (4) 5 (1972), 71-120.

2. Construction de représentations unitaires d'un groupe de Lie, Harmonic Analysis and Group Representations, C.I.M.E., Naples, 1980, pp. 129-221.

3. - Théorie de Mackey pour les groupes de Lie algèbres, Acta Math. 149 (1982), 153-213.

4. R. Howe, On the character of Weil's representation, Trans. Amer. Math. Soc. 177 (1973), 287298.

5. __ Topics in harmonic analysis on solvable algebraic groups, Pacific J. Math. 73 (1977), 383-435.

6. A. Kleppner and R. Lipsman, The Plancherel formula for group extensions, Ann. Sci. École Norm. Sup. (4) 5 (1972), 459-516; 6 (1973), 103-132. 
7. R. Lipsman, Orbit theory and harmonic analysis on Lie groups with co-compact nilradical, J. Math. Pures Appl. (9) 59 (1980), 337-374.

8. (9) 60 (1982), 17-39.

9. __ Harmonic induction on Lie groups, J. Reine Angew. Math. 344 (1983), 120-148.

10. $\ldots$, On the existence of a generalized Weil representation, Proc. Conf. on Group Representations (Marseille, 1982), Lecture Notes in Math., vol. 1020, pp. 161-178.

11., On the existence of metric polarizations, Rocky Mountain J. Math. (to appear).

12. G. Mackey, Unitary representations of group extensions, Acta Math. 99 (1958), 285-311.

13. R. Penney, Canonical objects in Kirillov theory on nilpotent Lie groups, Proc. Amer. Math. Soc. 66 (1977), 175-178.

14. W. Rossman, Kirillov's character formula for reductive Lie groups, Invent. Math. 48 (1978), 207220.

DePaRtMENT OF MATHEMATICS, UNIVERSity OF MARYLAND, COLlege PARK, MARYLAND 20742 